\title{
The design and realization of embedded electronic guide dogs
}

\author{
YUE Xiangyu \\ School of Management and Engineering, Nanjing University; Nanjing Jiangsu; 210008; PR China
}

Email address:

yuexiangyu168@gmail.com

\section{To cite this article:}

YUE Xiangyu. The Design and Realization of Embedded Electronic Guide Dogs. American Journal of Embedded Systems and Applications. Vol. 1, No. 1, 2013, pp. 13-16. doi: 10.11648/j.ajesa.20130101.12

\begin{abstract}
With the rapid development of embedded technology, a variety of embedded application systems gradually come into our lives. The design applies the embedded characteristics to the construction of the electronic guide dog system, meeting the agility needs of the electronic guide dogs. In order to make the existing network video surveillance system miniaturization and simplification, we propose a general webcam implementation. In the design, the embedded Linux operating system is transplanted into Samsung S3C2440 ARM9 chip. The design mainly uses the webcam as a sensor for obstacle avoidance and detection of moving objects, and makes the Web server miniaturization to save space and reduce costs. The system also uses the voice control Beidou navigation device, so the electronic guide dogs can be accepted the commands given by "dog-controlling man", position the destination automatically and accurately, and guide the "dog-controlling man" marching to it. The system also has good real-time nature and automatic interactivity, and it can be widely used in a variety of electronic guide dogs, so it has broad development prospects.
\end{abstract}

Keywords: Embedded, Electronic Guide Dog, Beidou Navigation Instrument, Webcam, S3C2440, Embedded Linux

With the rapid development of China's transport, the frequency of traffic accidents is also high. In particular, the accidents caused by the blindness happen more frequently. So the blind traffic safety issue has become a social problem to be solved urgently. The design of embedded electronic guide dog in the project has many advantages such as low cost, flexible movement, reliable use, etc. It can effectively eliminate the impact of traffic environment and the subjective factors of the blind, sending the pre-trip alarm in time, effectively avoiding the obstacles like vehicles, to reduce traffic accidents caused by the blindness. This humanized science and technology innovation has a positive role in promoting the development of Chinese transport and blind welfare.

\section{The Platform Architecture of the Embedded Electronic Guide Dog Hardware}

The embedded electronic guide dog system mainly is consisted of acoustic Beidou navigator, video collection and processing module, $3 \mathrm{G}$ wireless network card, embedded Web Server, and alarm system. The embedded Web Server includes the ARM processor S3C2440 and network chip of the Samsung. ${ }^{[1]}$ First of all, the blind gives instruction to the guide dog by sound, and tells it the destination. Then the guide dog may position automatically through the acoustic Beidou navigator, getting to the destination immediately. On its way, the guide dog transmits the image data, collected by camera to the video processor. After compressing and encoding the data, the video processor will transfer ARM processor. Then the ARM processor will compare the data of this frame with the previous frame. If the data is basically same, it indicates that there is no moving object, and then discards this frame to save storage space; but if there is a huge difference of the data, it indicates that there exists moving object. Then the processor would save this frame, and start the acousto-optic module immediately to call the police. The embedded electronic guide dog warning system elects USB protocol to transmit data. When alarming, it utilizes the combination of light and sound. Sound will produce auditory stimulus for the blind. Meanwhile, light and sound may make an auditory and visual stimulation for other pedestrians, arousing the attention of the blind and pedestrians to let them take appropriate actions at the same time to avoid accidents. If the client-side of the family member wants to know the blind's location and the 
surrounding safety situation in time, they could check the real-time video through Web server.

The system hardware of guide dog mainly includes acoustic Beidou navigator, ARM processor S3C2440 of Samsung, camera and image preprocessing chip, 3G wireless network card, and sound-light alarm module. The acoustic Beidou navigator could let the guide dog receive the voice commands of the blind to accurately locate the destination by the navigator. Then, the guide dog gets to the destination according to the instruction of the navigator. The 32-bit ARM processor realizes the caching architecture of MMU, AMBA BUS, and Harvard, owning the independent $16 \mathrm{~KB}$ instruction Cache and $16 \mathrm{~KB}$ data Cache. ${ }^{[2]}$ The network chip is the general CS8900. And the SDRAM is two pieces of HY57 V561620 (32M). The NORFLASH is AM29LV 160DB. NAND FLASH adopts the 32M K9F 1208 chip. Video processing selects the hardware H.264 codec, specifically the QL202B, which is the low-power dissipation real-time single-chip half duplex H.264/AVC. The hardware structure of the guide dog system is shown as Figure 1.

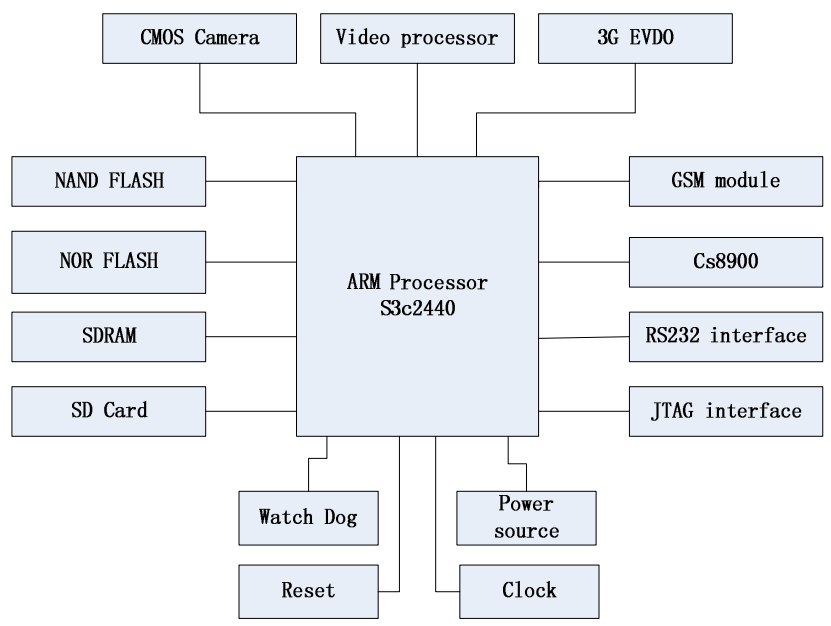

Figure 1: the hardware structure diagram of the guide dog system ${ }^{[3]}$

\section{The Design of Embedded Electronic Guide Dog Software}

The software design of embedded electronic guide dog system mainly includes the transplant of embedded Linux operating system, the writing of video collecting program and dynamic detecting program, the realization of embedded Web Server and the writing and transplant of its related drivers $^{[4]}$

\subsection{The Visual Dynamic Detection of the Guide Dog}

The visual dynamic detection of the guide dog refers to the dynamic detection of the video capture of the guide dog. Its principle is shown as the Figure 2. Firstly, it sets the period of the video acquisition cycle of the camera to $\mathrm{T}$, and save the obtained first frame of the image background after the system initialization. Then, it collects one frame of every interval $\mathrm{T}$, and tests the moving object by background subtraction. This approach is the real-time extraction of image data from the video stream. By comparing with each other, it will detect the related points of image sequence and the $3 \mathrm{D}$ objects, and then filter the irrelevant information of moving objects in the image. This software uses QT/E image processing library--QImage process to develop. It initially collects two image data by circle to carry on a binaryzation processing of the image, and then respectively extracts the brightness of the two images as difference. When getting the detail image of the background difference image, it immediately compares the image with the settled brightness threshold value. If it does not exceed the threshold, this frame should be discarded; if it is more than this threshold, then the alarm system should be triggered immediately.

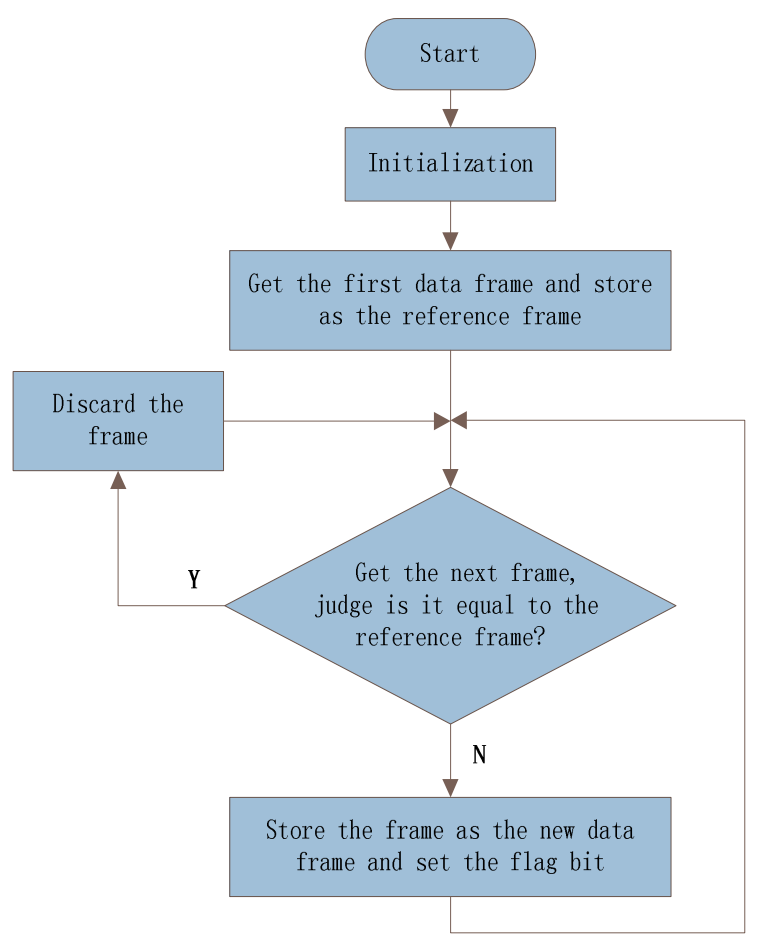

Figure 2: the visual dynamic detection flow of the guide dog ${ }^{[3]}$

\subsection{The Software Design of the Guide Dog Embedded Web Server}

The software of the guide dog embedded Web Server ${ }^{[5]}$ mainly includes two parts: (1) The transplant and configuration of Boa; (2) The embedded CGI. ${ }^{[6]}$ The workflow of the guide dog embedded Web is shown as the Figure 3.

\subsubsection{The Transplant and Configuration of Boa}

Boa is a single task Http server, which has excellent performance and fast speed. You could download a mainstream version of the boa distributions from http://www.boa.org, as well as the source code, and then start configuring and transplanting. The configuring and transplanting method of Boa could be checked easily on the Internet, so I won't reiterate it here. 


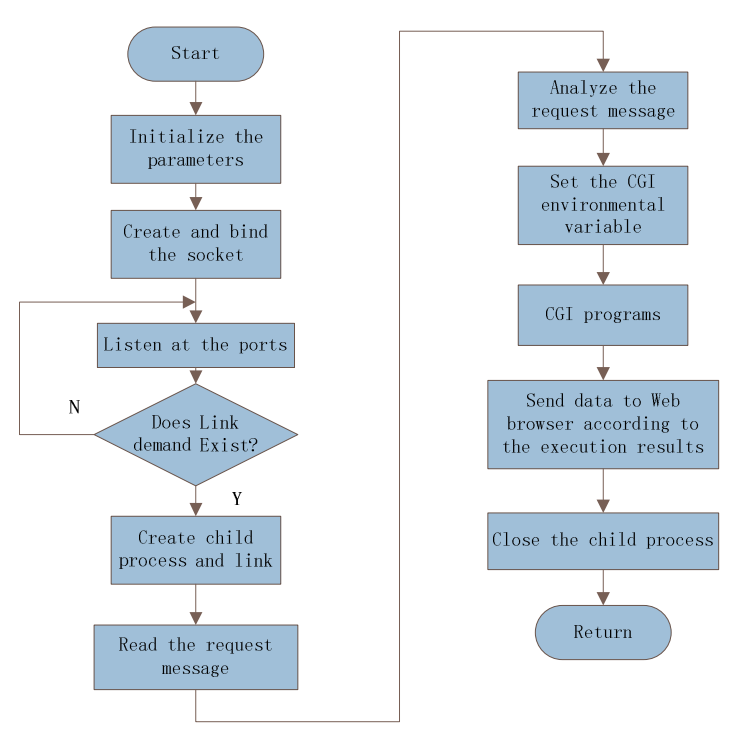

Figure 3: the embedded Web server workflow of the guide $\operatorname{dog}^{[3]}$

\subsubsection{The Embedded CGI}

The traditional website is static, not having the function of dynamic display and interactive operation. In order to solve this problem, we have introduced the CGI (Common Gateway Interface), realizing the dynamic display and interactive operation of Web. Embedded CGI is a standard interface for the interaction of the external expanding application and the WWW server, through which can achieve the interactive operation between Web client and server. The embedded CGI could provide the functions that static HTML webpage could not achieve, for example, search engine, remote control, database access based on Web data, and so on. In addition, it has some other advantages, including fast running speed, the support of various programming language, and browser capabilities. Of course, the browser here is not controlled by the hand of the blind, but controlled by the system itself and the voice of the blind. The process of realizing the interaction of client and server by the embedded CUI is shown in the Figure 4.

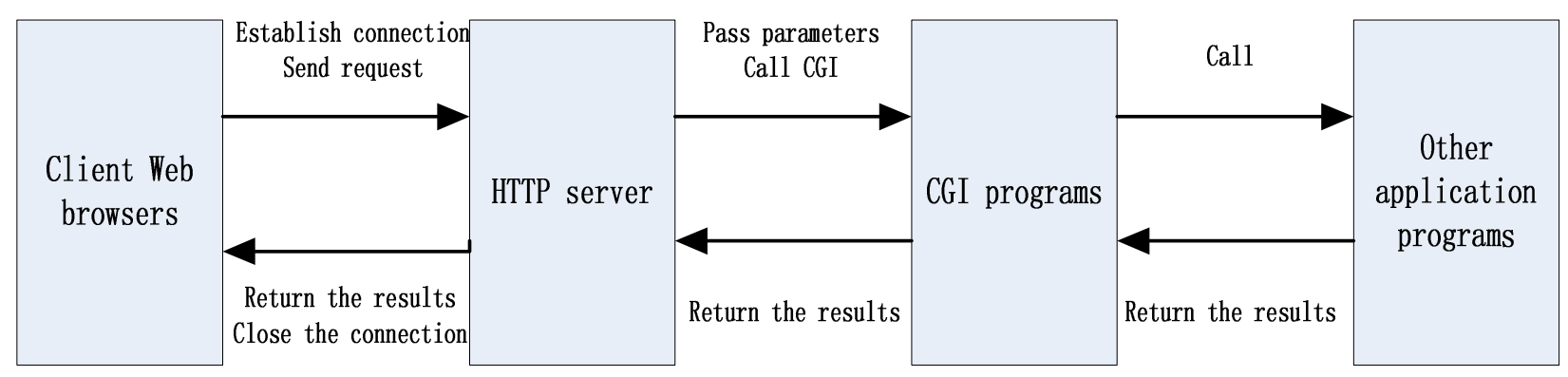

Figure 4: HTTP regulating and applying CGI process ${ }^{[3]}$

\section{Conclusion}

Embedded electronic guide dog system doesn't need a person to open the browser to input an IP number, but can obtain an IP address via voice, and automatically login embedded server. The embedded electronic guide dog system described by the author in this article has the advantage of ease of placement, so it has a very broad application prospects. However, it still needs to be perfected. For example, the capacity of the storage of the video is limited; Every "guide dog" has a separate IP, and it occupies much of IP resource comparatively; The amount of video information is very large; The transfer rate of $3 \mathrm{G}$ wireless network card is limited, and it has a few seconds delay in transmission. We believe that in the near future, with the development of the compression algorithm technology and $3 \mathrm{G}$ wireless network card transmission, the issues such as the popularization of IPV6 protocol will be resolved gradually.

\section{Brief Introduction to the Author}

Yue Xiangyu (1992- ), who is a male, Han nationality, was born in Weifang, Shandong, with BA, Nanjing
University, mainly engaged in electrical information and automation, aesthetics and other aspects of learning and research, specializing in modern industrial embedded control systems, networked control systems, intelligent control. He presided over one national college student science and technology innovation project, taking part in the research on one "Eleventh Five-Year Plan" education and science key project of the Education Ministry, and one soft science research project of Shandong Province. He has 12 science and technology papers published in Chinese and oversea academic journals. He has won the 1st scholarship of China, the 1st scholarship of people, the 1st and the 2nd prizes of China Education Robotics Competition, the 1st prize of China Mathematical Modeling Contest, the top award of scientific research achievement of Nanjing University, two China science and technology patents and the honorary title, "three-good-student" of Jiangsu Province. In addition, as a representative of Nanjing University, he went to the National University of Singapore to participate in an academic exchange program. Address: School of Management and Engineering, Xianlin Campus of Nanjing University, 163, Xianlin Avenue, Qixia District, Nanjing, Jiangsu Province, PR China; Zip: 210008. 


\section{References}

[1] Zhou Xiaoguang, Pan Yantao. S3C2440A-based embedded video system design[A]. Electronic Measurement Technology[J], 2006, 29 (6): 89-91.

[2] Wang Peizhen, Xu Junsheng. ARM9-based embedded Linux Image Acquisition System design[A]. Intelligent control, detection technology and its application [J], 2007, 36 (19): 89-92.

[3] Guo Changdong, Yao Shuncai. Car Security and Defence

System Design and Implementation[A]. Electronic Component Application J], 2011, (3).

[4] Wang Jianfei, Meng Yanjie, Zhao Yong. The video capture card driver design of Linux-based operating system[A]. Microcomputer Information[J], 2007, (2): 102-104.

[5] Jiang Min, Shi Jian. The remote video surveillance technology based on embedded WEB server[A]. Computer age [J], 2007 (5): 31-32.

[6] Ye Weiqiong. The Implementation Method of Embedded Web Server intelligent home central controller [M]. Guangzhou: Guangdong University Press, 2005. 